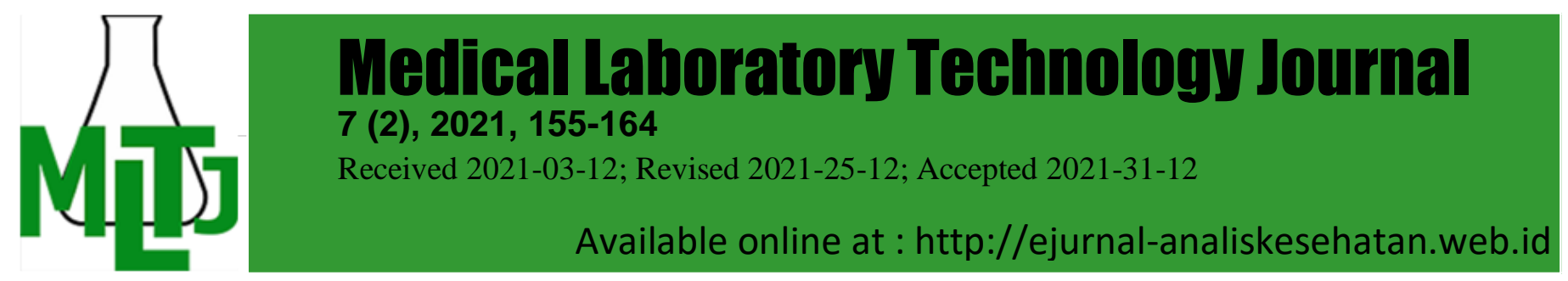

\title{
Effectiveness of Boiling Method Using Aquadest Solvent in Oolong Tea Extraction Producing Mangenese Particle as a Contrast Media on Magnetic Resonance Cholangiopancreatography (MRCP)
}

\author{
${ }^{*}$ Fatimah Fatimah ${ }^{1}$, Neni Susilaningsih ${ }^{1}$, Hermina Sukmaningtyas ${ }^{1}$, \\ Agus Subagio ${ }^{2}$
}

${ }^{1}$ Faculty of Medicine, Diponegoro University, Indonesia, ${ }^{2}$ Physics Department Technology Faculty, Diponegoro University, Indonesia.

*Email: fatimah_yunaeza@yahoo.com

DOI: $10.31964 / \mathrm{mltj} . v 7 i 2.423$

\begin{abstract}
Magnetic Resonance Cholangiography (MRCP) examines the pancreas and ductus billiaris using magnetic resonance imaging with a negative contrast media administered orally. Oolong tea is possible to be an alternative of an oral negative contrast media due to its manganese contained. This study will elaborate on the best method of oolong tea extraction to get the best mangenese substance and its capability to suppress inevitable organs. This is an experimental study with various methods of extraction, which are the maceration method with ethanol solvent, maceration method with aquadest solvent, and boiling method. The whole process includes collecting and preparing plant materials, sample identification, sample processing, extraction, and extract characterization. The manganese level is checked with Atomic Absorption Spectroscopy (AAS) followed by scanning the extracted oolong tea in bottles and MRCP scanning examination. Results showed that manganese level with three different extraction methods is maceration method with ethanol is $1.23 \mathrm{mg} / \mathrm{dl}$, maceration method with aquadest solvent is $0.82 \mathrm{mg} / \mathrm{dl}$, and the extraction using the boiling method with aquadest solvent $0.94 \mathrm{mg} / \mathrm{dl}$, and manufactured tea $1.40 \mathrm{mg} / \mathrm{dl}$ respectively. Scanning the extracted oolong tea in bottles showed that the best suppression is on the bottle, which contains extracted oolong tea using a boiling method with aquadest solvent. The image enhancement on MRCP showed that biliary trees are all enhanced using all the extraction methods; however, the best suppression for the stomach and duodenum is using the boiling method with aquadest solvent. The best choice for oolong tea extraction to get the best image to enhance and maximum suppression for disturbing organs is the boiling method with aquadest solvent. For the oolong tea, oral media contrast is to consider the level of manganese substance and its capability to suppress the disturbing organ to provide the best image for MRCP.
\end{abstract}

Keywords: oolong tea; boiling method extraction; manganese; magnetic resonance cholangiopancreatography

\section{INTRODUCTION}

Oral MRI contrast media, according to their role in generating signal intensity, were grouped into positive contrast media and negative contrast media. Positive contrast media can increase the signal intensity, while negative contrast media helps suppress the signal intensity of a particular tissue ( $F$. Fatimah, Sugiyanto, \& Wibowo, 2016). Some examples of negative oral contrast media used to examine the abdomen are Gadopentetate dimeglumine, Ferric ammonium citrate, Manganese chloride, Kaolinite, antacids, Barium sulfate, and Ferric particles. Currently, negative 
oral contrast media are rarely used because they are no longer produced, have a bad taste, are very difficult to swallow, and are relatively expensive (Riordan, Khonsari, Jeffries, Maskell, \& Cook, 2004).

Several studies to find alternatives to natural negative oral contrast media in MRCP examination have been carried out, in the form of liquid or juice, both in fruit and tea drinks (Prasert, Rugkhasukon, \& Tanomkiat, 2009). The fruits that have been used are blueberries (Hiraishi et al., 1995; Papanikolaou, Karantanas, Maris, \& Gourtsoyiannis, 2000), pineapple (Riordan et al., 2004), blackberries (Espinosa et al., 2006), and lemon or orange (Chu, Ji, \& Zhang, 2010). In this study, it was found that blueberry juice contains a lot of Manganese $(\mathrm{Mn})$, which functions as a positive contrast medium in T1-weighted images to maximize gallbladder and bile duct visualization, as well as a negative contrast medium in T2-weighted images to suppress signals in the stomach and duodenum. Blueberry juice is tough to find, especially in tropical countries like Indonesia. Some researchers have suggested using another alternative, namely pineapple juice. Pineapple juice has the same function as blueberries but has a sour taste; sometimes patients refuse to drink it, while blackberry juice contains a lot of Ferrum $(\mathrm{Fe})$, which is more sensitive for liver $\mathrm{MRI}$ examination, so it is not widely used for MRCP examination.

Oolong tea comes from Camellia Sinensis, basically made from the same type of tea leaves as green tea and black tea. One of the benefits of oolong tea is that it can prevent flatulence due to excess stomach acid production and protect the stomach wall because it contains the antioxidant L-theanine (Alamsyah, 2006). Oolong tea with a manganese content of $0.90 \mathrm{mg}$ with a volume of $400 \mathrm{ml}$ has been researched on its use as an alternative to natural negative oral contrast media in MRCP examination, and the best scanning start time is 15 minutes post-contrast natural negative oral oolong tea (A. S. Fatimah \& Sugiyanto, 2018). Several previous studies have shown that the best time to start scanning is 15 minutes after using natural negative oral contrast media (Ghanaati et al., 2011). Furthermore, giving the volume of tea liquid about $300 \mathrm{ml}-500 \mathrm{ml}$ becomes a separate obstacle in showing it because it is too much for a one-time serving.

Based on some of the research limitations mentioned above, when applied to patients with pathological conditions, it will be difficult to take large amounts of oral contrast media (>200 ml). In some cases, suppression or suppression of stomach signal intensity is not maximal, so it is still possible that there are pathological conditions that cannot be visualized optimally. In addition, in general, the scanning start time is relatively long to produce maximum image intensity.

This study intends to provide an alternative oolong tea contrast media for MRCP with a higher level of manganese and optimal diagnostic images of the pancreas and biliary trees using a lesser volume of oolong tea contrast media administered orally. Oolong tea as an oral negative contrast media for MRI is considered acceptable taste, easy to obtain in the territory of Indonesia, safe for consumption. It has an optimal and short scanning start time.

\section{MATERIALS AND METHODS Oolong Tea Extraction Methods}

The plant used is Camellia Sinensis and is found in Kemuning Village, Central Java, Indonesia. The process of extracting oolong tea leaves was conducted by maceration, namely: $100 \mathrm{~g}$ of Simplicia powder of oolong tea leaves each was put into a glass container; firstly, it must be macerated with $1 \mathrm{~L}$ of $96 \%$ ethanol solvent and 1 $L$ of distilled water; when the process was finished it was covered and leave it for two 
days. While protected from the light, it frequently stirred, shredded, squeezed, washed the dregs with enough filter liquid to obtain $1 \mathrm{~L}$. Then it was transferred to a closed vessel and leave it in a cool place. It must be protected from light for two days, and the second process must be bowled with boiling at a temperature $140^{\circ} \mathrm{C}$ for 5 minutes then at $100^{\circ} \mathrm{C}$ for $15-20$ minutes using aquadest solvent. The results obtained were concentrated with a rotary evaporator type Rotavapor® R-100 until most of the solvent had evaporated. The evaporation process was continued on a water tube until a thick extract was obtained.

In addition to this extraction method, the extraction product from maceration using ethanol solvent can be made as a powder using a freeze dryer type Alpha 1-2 LDPlus. The $100 \mathrm{~g}$ of oolong tea was dissolved in $1 \mathrm{~L}$ of ethanol then mixed into an ultaturax type IKA Ultra Turrax T25 at a speed of 1500-2000 RPM for 2 hours.

This is an experimental research design. The whole process included the collection and preparation of oolong tea leaves materials, sample identification, sample processing, extract preparation, extract characterization, and analysis for the determination of manganese elements using an Atomic Absorption Spectroscopy (AAS) type novAA 300.

\section{MRI Scanning Process on Bottles and Volunteers}

The research subjects were $15 \mathrm{ml}$ of oolong tea extract dissolved in $200 \mathrm{ml}$ of distilled water for MRCP examination using an MRI machine with Magnetom Avanto Eco 1,5 Tesla. First, a scanning test using an MRI scan was carried out on seven bottles with a volume of $200 \mathrm{ml}$ consisting of $185 \mathrm{ml}$ of distilled water and $15 \mathrm{ml}$ of oolong tea extracted from different methods. The seven bottles contain are 1) a bottle included with an extracted oolong tea using maceration method with ethanol solvent, 2) a bottle with extracted oolong tea with a boiling method with aquadest solvent, 3) a bottle contained with an ultaturax method with ethanol solvent, 4) a bottle contained with oolong tea using maceration method with aquadest solvent, 5) a bottle contained with oolong tea from tea brand $X$ packaged, 6) a bottle contained with aquadest, 7) a bottle contained with oolong tea from tea brand $Y$ packaged.

Then the MRCP examination was carried out on two volunteers who administered orally $200 \mathrm{ml}$ of extracted oolong tea of the two highest manganese levels respectively. MRI machine specification and parameters were the same between the bottle scanning and volunteers scanning. Parameter of MRI scanning for both bottles and MRCP scanning were T2 HASTE Fat Saturation Coronal Thick Slab Radial. Assessment of MRCP image information was carried out by assessing the signal-to-noise ratio on negative oral images of the effects of contrast media on the pancreaticobiliary tree image system. This study is permitted by the Ethics Committee of Kariadi General Hospital Semarang, Central Java.

\section{RESULTS AND DISCUSSION}

\section{Manganese Level of Various Methods of Oolong Tea Extraction}

After finished the extraction method, the level of manganese of the extraction products was analyzed using AAS. As a control, this study also analyzed the manganese level of band $X$ and $Y$ of oolong tea that already exists in the market as a bottled drink and the level of manganese of the aquadest. The varies of manganese levels from different methods and products can be seen in Table.1. The results of Atomic Absorption Spectroscopy (AAS) are as follows: 


\begin{tabular}{|c|c|c|}
\hline No & Varies of Process & $\mathrm{mg} / \mathrm{dL}$ \\
\hline 1 & $\begin{array}{l}\text { Maceration method with } \\
\text { Ethanol solvent }\end{array}$ & 1.23 \\
\hline 2 & $\begin{array}{l}\text { Boiling method with Aquadest } \\
\text { solvent }\end{array}$ & 0.94 \\
\hline 3 & $\begin{array}{l}\text { Ultaturax method with Ethanol } \\
\text { solvent }\end{array}$ & 0.37 \\
\hline 4 & $\begin{array}{l}\text { Maceration method } \\
\text { Aquadest solvent }\end{array}$ & 0.82 \\
\hline 5 & Tea brand $X$ packaged & 0.14 \\
\hline 6 & Aquadest & 0.00 \\
\hline 7 & Tea brand $Y$ packaged & 0.14 \\
\hline
\end{tabular}

From table 1. it can be seen that the highest manganese level is produced by the oolong tea extraction using maceration with ethanol solvent $(1.23 \mathrm{mg} / \mathrm{dL})$, while the extraction method using a boiling with aquadest solvent is produced $0.94 \mathrm{mg} / \mathrm{dL}$, and an extraction using maceration with aquadest solvent is $0.82 \mathrm{mg} / \mathrm{dL}$. In table 1 , it is also seen that the manganese level of the other products is very low.

\section{MRI Scanning on the Bottles Contained Variation Oolong Tea Products}

The next step was to conduct an MRI scanning for all the products in the bottle each. Due to the negative oral media contrast is commonly scanned using the T2 weighted images with HASTE (half Fourier acquisition single-shot TSE) fat saturation to detect the suppression effect of gastrointestinal organs surrounding pancreas and biliary trees. MRI parameters scanning for bottles is TR (time repetition) $4500 \mathrm{~ms}$, TE (time echo) $700 \mathrm{~ms}$, ETL (echo train length ) 300, average 1, slice thickness $50 \mathrm{~mm}$, scan time $50 \mathrm{sec}$. The bottles scanning also uses the same scanning parameter as MRCP scanning (Griffin, Charles-Edwards, \& Grant, 2012). The result of MRI scanning can be seen in the following image (fig. 1) :

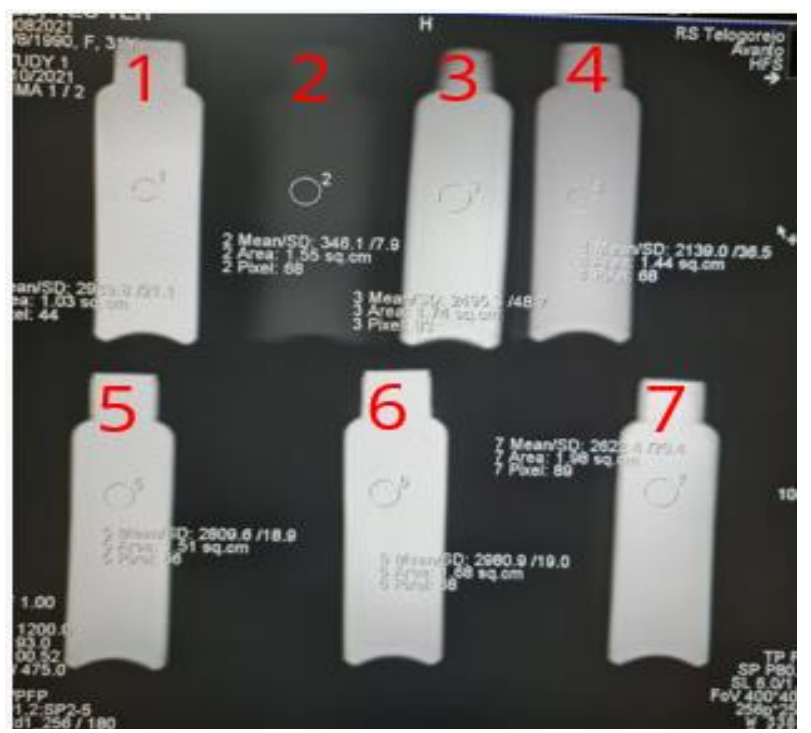

Figure 1. MRI Image from the Scanning on the Bottles with the Various Product of Extraction 1) Maceration Method with Ethanol Solvent, 2) Boiling Method with

Aquadest Solvent, 3) Ultaturax Method with Ethanol Solvent, 4) Maceration Method with Aquadest Solvent, 5) Tea Brand X Packaged, 6) Aquadest, 7) Tea Brand Y Packaged. 
From the results of these images in Figure 1, it can be seen that not all of the pictures of the bottle are suppressed with the T2 HASTE fat saturation scanning. The best suppression is detected on the bottle of number 2 , which is dark on the image, the oolong tea extracted using boiling with aquadest. The lesser suppression is also detected on the bottle of numbers 3 and 4, the oolong tea extracted with ultaturax method with ethanol solvent and maceration method with aquadest solvent, respectively. While the other bottles are detected with strong signals, they look white on the image, which are the bottle of numbers 1 , number 5,6 , and 7 . The bottle of number 1 is the oolong tea extracted using maceration with ethanol solvent with the highest manganese level based on AAS analysis.

\section{MRCP scanning on the volunteers}

The next step is to administer the extracted oolong tea to the volunteers. In this study, choose the two highest manganese levels of the extracted oolong tea, which are the oolong tea extracted using ethanol solvent and the oolong tea extracted using boiling with aquadest solvent. Each volunteer drinks $215 \mathrm{ml}$ oolong tea extracted using maceration with ethanol solvent and oolong tea extracted with boiling using aquadest solvent. The MRCP scanning is conducted before and ten minutes after oolong tea administration. The scanning parameter is the same as the scanning parameter on the bottle scanning. The result of the MRCP scanning before (pre) and after (post) administration of oolong tea can be seen in figures number 2 and 3 .

To see the effect of the image of the manganese content of oolong tea extraction on the MRCP examination, an assessment will be carried out; (a) on the ability to suppress the signal intensity of the stomach, duodenum, liver, and pancreas, (b) on the ability to amplify the signal intensity of the pancreaticobiliary tree system; both are measured using localization in the Region of Interest (ROI) to get the Signal to Noise Ratio (SNR) value.

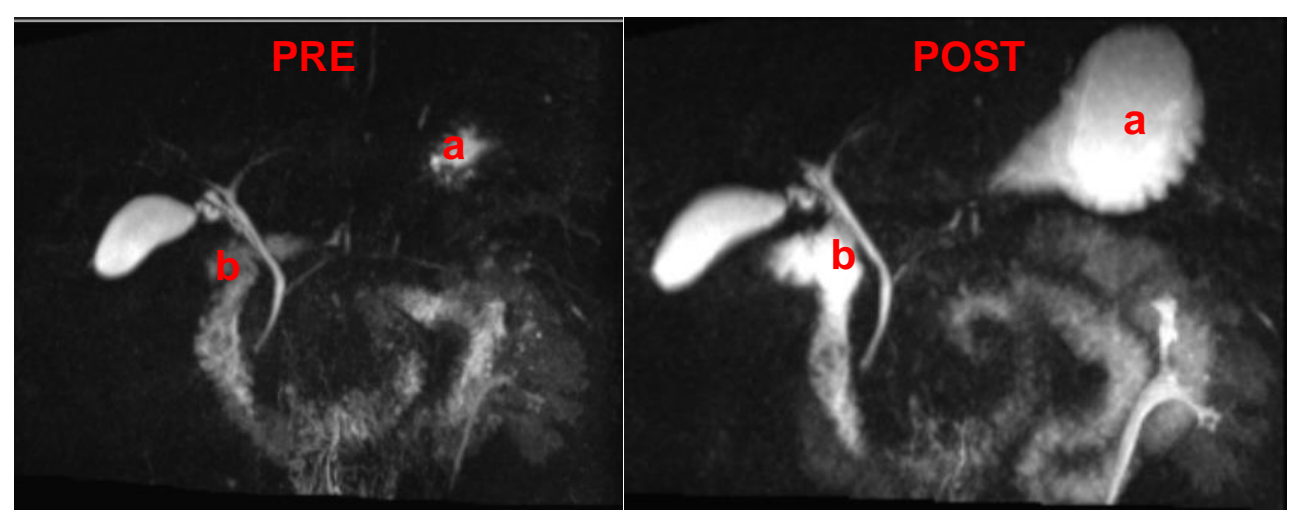

Figure 2. Manganese from Maceration Extraction Method with Ethanol Solvent. MRCP Image of Organ Stomach (a) and Duodenum (b) in Pre and Post-contrast are not Suppressed. 


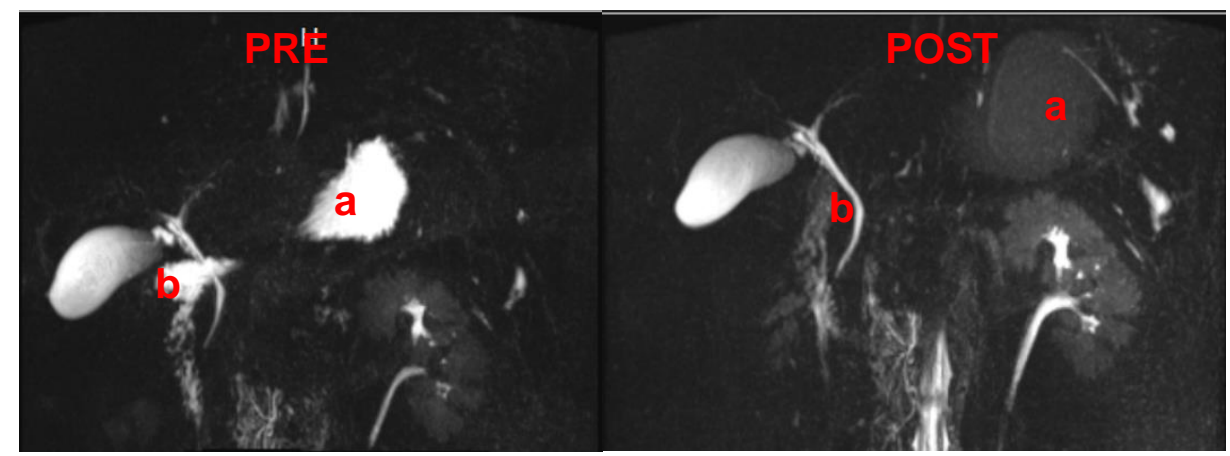

Figure 3. Manganese from Boiling Extraction Method with Aquadest Solvent. MRCP Images of Organ Stomach (a) and Duodenum (b) are Suppressed

Post-Contrast.

To determine the signal level of the gall bladder, biliary trees and gastrointestinal (stomach and duodenum) are measured its signal to noise ratio (SNR). The value of SNR can be calculated using software in the MRI machine by making a circular ROI (region of interest) on each organ. The SNR value of MRCP images at pre and post-administration oolong tea can be seen in Tables 2 and 3.

Table 2. SNR Value on MRCP Images at Pre and Post Administration of Oolong Tea Extracted by Maceration Method with Ethanol Solvent

\begin{tabular}{llll}
\hline \multirow{2}{*}{ No } & \multirow{2}{*}{ Organ } & SNR & \\
\cline { 3 - 4 } & & PRE & POST \\
\hline 1 & Stomach & 27.98 & 27.8 \\
2 & Duodenum & 14.47 & 37.94 \\
3 & Gall Bladder (GB) & 32.9 & 35.25 \\
4 & Cystic Duct (CD) & 27.58 & 17.46 \\
5 & Common Hepatic Duct (CHD) & 24.17 & 22.94 \\
6 & Intra Hepatic Duct (IHD) & 5.58 & 7.21 \\
7 & Cammon Bile Duct (CBD) & 24.03 & 26.7 \\
8 & Pancreatic Duct (PD) & 7.43 & 7.82 \\
\hline
\end{tabular}

In table 2. It is found that the SNR value of each organ varies. On the preadministration of oolong tea extracted by maceration using ethanol solvent, the high SNR showed at the organ of gall bladder (32.9), stomach (27.98), cystic duct (27.58), common hepatic duct (24.17), and common bile duct (24.03). On the other hand, on the post administration of oolong tea extracted by maceration using ethanol duodenum (37.94), gall bladder (35.25), stomach (27.8), common bile duct (26.7), common hepatic duct (22.94) showed the high SNR. Dramatic SNR change between pre and post-administration oolong tea is shown on the duodenum (increasing), cystic duct (decreasing), while on the stomach, gall bladder, common hepatic duct, and common bile duct are relatively the same. 
Table 3. SNR Value on MRCP Images at Pre and Post Administration of Oolong Tea Extracted by Boiling Method with Aquadest Solvent

\begin{tabular}{llll}
\hline \multirow{2}{*}{ No } & \multirow{2}{*}{ Organ } & SNR & \\
\cline { 3 - 4 } & PRE & POST \\
\hline 1 & Stomach & 44.29 & 4.27 \\
2 & Duodenum & 36.94 & 3.08 \\
3 & Gall Bladder (GB) & 32.6 & 33.17 \\
4 & Cystic Duct (CD) & 12.06 & 11.86 \\
5 & Common Hepatic Duct (CHD) & 27.52 & 20.83 \\
6 & Intra Hepatic Duct (IHD) & 6.79 & 4.99 \\
7 & Cammon Bile Duct (CBD) & 19.65 & 20.07 \\
8 & Pancreatic Duct (PD) & 3.3 & 3.42 \\
\hline
\end{tabular}

In table 3. shows that on pre and post administration of oolong tea extracted using boiling with aquadest solvent organ of the stomach and duodenum, decreasing its SNR drastically, while other organs on the gall bladder, cystic duct, common bile duct are relatively the same in high SNR; however, in the intrahepatic duct and pancreatic duct is relatively the same at the low SNR.

Several studies have been conducted to find alternatives of a natural negative oral contrast media in the MRCP examination. Wether in any kind of fruits such as blueberry (Papanikolaou et al., 2000) (3-4 mg/dl), pineapple (Riordan et al., 2004) $(0.276 \mathrm{mg} / \mathrm{dl})$, blackberry $(2.93 \mathrm{mg} / \mathrm{dl})$, lemon or orange (Chu et al., 2010$)(0.2 \mathrm{mg} / \mathrm{dl})$ or black tea (Ghanaati et al., 2011) $(0.44 \mathrm{mg} / \mathrm{dl})$. The main reason is that fruits or tea contain a lot of manganese. For a negative contrast media in MRCP scanning, manganese material has an important role in suppressing a disturbing organ in the targeted organ. On MRCP examination, the targeted organs that must be detected are the gall bladder and biliary trees, while the disturbing organs are on gastroduodenal such as stomach and duodenum. These organs are usually overlapping with the targeted organ, which can cause misdiagnosis.

The previous study (A. S. Fatimah \& Sugiyanto, 2018) showed that oolong tea is used as an alternative negative contrast media in MRCP examination due to its high manganese content $(0.9 \mathrm{mg} / \mathrm{dL})$ compared to other kinds of teas. That study proved that oolong tea increases the contrast between the biliary trees images and surrounding organs. However, the suppression to the stomach and duodenum was not optimal subsequently. It may cause misdiagnosis of the pathological condition in the pancreaticobiliary organ. This study rules out such material of oolong tea that can increase its suppression to the stomach and duodenum. It's a relatively safe and acceptable taste to the patient.

There are many kinds of oral negative contrast media used for abdominal examination in MRI, such as gadopentetate dimeglumine, ferric ammonium citrate, manganese chloride, kaolinite, antacid, ferristene, ferumoxil, perfluoro-octyl bromide, and ferric particles. Currently, these negative contrast media are made of $\mathrm{Mn2}+$ or $\mathrm{MnCl} 2$ ions (0.5-1 mg/dl Mn-DTPA), some of which are rarely used because they are no longer produced, have a bad taste, are very difficult to swallow, and are relatively expensive (Bae et al., 2012; Pressacco et al., 2013). In addition, some contrast media is administered intravenously. The level of cellular toxicity is higher than oral administration; the short plasma half-life is very short, so it isn't easy to collect in the blood pool agent, and there is still retention in the basal ganglia tissue for a long examination. Contrast media such as mangafodipir trisodium, used as a negative 
intravenous contrast medium, showed biodistribution in mice at 30 minutes after injection that $13 \%$ was in the liver, $9 \%$ in the small intestine, $3 \%$ in the blood, and $1.3 \%$ in the intestine. Kidney (Regge, Cirillo, Macera, \& Galatola, 2009). The overall difference in MRCP image information both on the effect of negative oral contrast media on stomach and duodenal signal intensity and on the effect of the image of the pancreaticobiliary tree indicates that oolong tea can be used as a negative substitute.

Table 1. showed that the highest manganese level is produced by the oolong tea extraction method using ethanol solvent $(1.23 \mathrm{mg} / \mathrm{dl})$. However, the bottle scanning (fig. 1) and volunteer scanning (fig. 2 and 3 ) showed that this extraction produces high signal intensity in pre and post-contrast media administration. It means that this oolong tea extracted with maceration using ethanol solvent does not meet the requirement of the negative oral contrast media in MRI, wherein negative oral contrast must suppress gastrointestinal signal on the T2 HASTE image. The oolong tea produced in this extracted with maceration using ethanol solvent tend to increase signal intensity which is a similar characteristic to the positive contrast media in MRI. The stomach and duodenum tend to increase their signals instead of suppressing them. Based on this result, although its manganese level is very high, it can not use as an alternative to oral negative contrast media in MRCP.

On the other hand, the oolong tea extracted with aquadest solvent shows a better image in suppressing both in the bottles (no 2) and the volunteers, although its manganese level is around $0.9 \mathrm{mg} / \mathrm{dL}$. It's lesser than oolong tea produced by maceration using ethanol solvent.

Figure 3 shows that the given the administration of oolong tea extracted using the boiling method with aquadest solvent, there is a difference in the stomach and duodenum between pre and post-contrast media administration of oolong tea. The stomach and duodenum are drastically decreasing their signal. This kind of tea can suppress the stomach and duodenum signal intensity. This is relevant to another previous study by (Riordan et al., 2004) and (Ghanaati et al., 2011).

Oolong tea extracted using boiling aquadest solvent can give a good image effect on the system of the pancreaticobiliary tree, namely the common bile duct and hepatic duct (Fig. 3). The intensity of this section indicates a difference in the effect of both the manganese from the Boiling extraction method with aquadest pre and post-contrast media administration.

The gallbladder and intrahepatic duct views are seen before or after administration of oolong tea contrast media, but there was no difference in the image effect. The presentation of oolong tea contrast media is considered less able to distinguish enlarged gallbladder and intrahepatic ducts on pre-contrast media in boiling extraction method with aquadest and oolong tea extraction using maceration with ethanol solvent from post-contrast media. The intrahepatic ducts can only be seen in the right and left hepatic duct branches, while the peripheral intrahepatic ducts are barely visible due to their smaller size, which agrees with previous findings (Vitellas, Keogan, Spritzer, \& Nelson, 2000).

The pancreatic duct and ampulla are barely visible on all MRCP images. The pancreatic duct is tilted slightly so that it is difficult to see the entire pancreatic duct. The use of a single Shot Fast Spin Echo (SSFSE) sequence will show pancreatic ducts in the head (97\%), body (97\%), and tail (83\%) (Vitellas, 2000). The fasting preparation aims to reduce gastric fluid. It is hoped that there will be a small amount of residual liquid in the second part of the duodenum, which is useful as a marker for the distal bile duct and ampulla. Administration of negative oral contrast media helps suppress 
signal intensity in the gut, but the ampulla is often not visible due to regurgitation of the contrast medium into the ampulla (Riordan et al., 2004).

The limitation of this study is the scanning time. The volunteers must hold their breath during the scanning to avoid any movement of the patient that will affect to images. In this study, it has been chosen the best parameter to get the best scanning time for the MRCP examination is 50 seconds; however, in any circumstances, the volunteers fail to hold their breath. If it happens, the scan must be repeated from the beginning to get the best images.

\section{CONCLUSION}

This study indicates that the ethanolic extract of cowpea (Vigna unguiculata) has the potential as an antioxidant to reduce serum MDA levels and prevent a decrease in the number of aortic endothelial cells and reduce brain MDA. As prevention, cowpea extract can be used as an antioxidant and consumed since premenopause to minimize problems that occur during postmenopause.

\section{CONFLICT OF INTEREST}

The authors have nothing to disclose.

\section{REFERENCES}

Alamsyah, A. N. J. P. A. M. P. J. (2006). Taklukkan penyakit dengan teh hijau: PT. ArgoMedia Pustaka.

Bae, K., Na, J., Choi, D., Cho, J., Choi, H., Jeon, K., . . . Chung, S. J. T. B. j. o. r. (2012). Contrast-enhanced MR cholangiography: comparison of Gd-EOBDTPA and Mn-DPDP in healthy volunteers. The British Journal of Radiology, 85(1017), 1250-1254.

Chu, Z.-Q., Ji, Q., \& Zhang, J.-L. J. A. i. (2010). Orally administered lemon/orange juice improved MRCP imaging of pancreatic ducts. Abdominal imaging, 35(3), 367-371.

Espinosa, M. G., Sosa, M., De León-Rodríguez, L. M., Córdova, T., Bernal-Alvarado, J., Avila-Rodríguez, M., . . Barrios, F. A. J. M. r. i. (2006). Blackberry (Rubus spp.): a pH-dependent oral contrast medium for gastrointestinal tract images by magnetic resonance imaging. Magnetic resonance imaging, 24(2), 195-200.

Fatimah, A. S., \& Sugiyanto, I. R. J. I. J. o. P. H. (2018). Oolong Tea Drink as an Alternative to Oral Negative Contrast Media in Magnetic Resonance Cholangio Pancreatography (MRCP). Indian Journal of Public Health, 9(9), 225.

Fatimah, F., Sugiyanto, S., \& Wibowo, G. M. J. L. (2016). Pendampingan Peningkatan Optimisasi Citra Mrcp Dengan Penggunaan Teh Oolong Dan Susu Soya Herbal Sebagai Alternatif Media Kontras Negatif Oral Di RS Kanker Ken Saras Ungaran Kabupaten Semarang. LINK, 12(1), 20-25.

Ghanaati, H., Rokni-Yazdi, H., Jalali, A. H., Abahashemi, F., Shakiba, M., \& Firouznia, K. J. E. r. (2011). Improvement of MR cholangiopancreatography (MRCP) images after black tea consumption. European radiology, 21(12), 2551-2557.

Griffin, N., Charles-Edwards, G., \& Grant, L. A. J. I. i. i. (2012). Magnetic resonance cholangiopancreatography: the ABC of MRCP. Insights into imaging, 3(1), 1121.

Hiraishi, K., Narabayashi, I., Fujita, O., Yamamoto, K., Sagami, A., Hisada, Y., . . . Hasegawa, H. J. R. (1995). Blueberry juice: preliminary evaluation as an oral contrast agent in gastrointestinal MR imaging. Radiological Society of North America, 194(1), 119-123. 
Papanikolaou, N., Karantanas, A., Maris, T., \& Gourtsoyiannis, N. J. J. o. c. a. t. (2000). MR cholangiopancreatography before and after oral blueberry juice administration. Journal of Computer Assisted Tomography, 24(2), 229-234.

Prasert, P., Rugkhasukon, S., \& Tanomkiat, W. J. S. M. J. (2009). The use of an iron or manganese enriched juice as a negative oral contrast agent in magnetic resonance cholangiopancreatography (MRCP). Songklandagarind Medical Journal, 27(3), 195-202.

Pressacco, J., Reinhold, C., Barkun, A., Barkun, J., Valois, E., \& Joseph, L. (2013). Accuracy of MRCP vs. ERCP in the Evaluation of Patients with Bile Duct Obstruction in the Setting of a Randomized Clinical Trial. Paper presented at the Proc. Intl. Soc. Mag. Reson. Med.

Regge, D., Cirillo, S., Macera, A., \& Galatola, G. J. R. i. M. I. (2009). Mangafodipir trisodium: review of its use as an injectable contrast medium for magnetic resonance imaging. Reports in Medical Imaging, 2(1), 55-68.

Riordan, R., Khonsari, M., Jeffries, J., Maskell, G., \& Cook, P. J. T. B. j. o. r. (2004). Pineapple juice as a negative oral contrast agent in magnetic resonance cholangiopancreatography: a preliminary evaluation. The British Journal of Radiology, 77(924), 991-999.

Vitellas, K. M., Keogan, M. T., Spritzer, C. E., \& Nelson, R. C. J. R. (2000). MR cholangiopancreatography of bile and pancreatic duct abnormalities with emphasis on the single-shot fast spin-echo technique. Radiographics, 20(4), 939-957. 